\title{
Iatrogenic Milk Alkali Syndrome in a Patient Recovering from Acute Renal Failure
}

\author{
Anum Fayyaz, R. Hal Scofield ${ }^{*}$ \\ Arthritis \& Clinical Immunology Program, Oklahoma Medical Research Foundation; Department of Medicine, College of Medicine, University of Oklahoma \\ Health Sciences Center, Medical Service, US Department of Veterans Affairs Medical Center, Oklahoma City, OK, USA
}

\begin{abstract}
Received: August 06, 2016; Accepted: August 30, 2016; Published: September 08, 2016
*Corresponding author: R. Hal Scofield, Department of Medicine, University of Oklahoma Health Sciences Center, Member, Arthritis \& Clinical Immunology Program, Oklahoma Medical Research Foundation, Staff Physician, Medical Service, Department of Veterans Affairs Medical Center, Oklahoma City, Oklahoma, USA, Tel: +1-405-271-7774; Fax: +1-405-271-4110; E-mail: hal-scofield@omrf.ouhsc.edu
\end{abstract}

\begin{abstract}
We describe a 49 year old morbidly obese man with iatrogenic milk alkali syndrome. He presented with sepsis resulting in acute renal failure with resultant hyperphosphatemia treated with high dose calcium carbonate. As his renal function improved, the calcium carbonate was continued and he developed high serum calcium with low serum parathyroid hormone. Serum calcium returned to normal with cessation of the calcium carbonate. We performed an extensive literature search that found many case reports and case series recognizing milk alkali syndrome as a common diagnosis of hypercalcemia on presentation. However, hypercalcemia was not a manifestation in our patient on presentation. There is scant literature on the subject of iatrogenic milk alkali syndrome. This complication in a hospitalized patient emphasizes that vigilance is required by the physicians while managing patients with multiple comorbidities, including chronic kidney disease, and numerous medications.
\end{abstract}

\section{Case Report}

A 49 year old morbidly obese man, with an extensive past medical history, presented to the emergency department with progressive shortness of breath, and subjective febrile episodes accompanied by chills. He had no complaints of chest pain, diaphoresis, nausea, vomiting, cough, abdominal pain and had negative history related to travel or any unusual immobility. He was hypotensive with a blood pressure of 74/65 $\mathrm{mmHg}$, pulse of $103 \mathrm{bmp}$, was breathing at 28 per minute, and his recorded temperature was $99.2 \mathrm{~F}$. His past medical history included of type 2 diabetes mellitus, hypertension, and obstructive sleep apnea with tracheostomy and stage 2 Chronic Kidney Disease (CKD) with a baseline serum creatinine of $2.4 \mathrm{mg} / \mathrm{dl}$. He was admitted with the diagnosis of septic shock supplemented by laboratory evidence of multiple organ failure. He was started on isotonic intravenous fluids along with ceftriaxone and vancomycin after blood cultures were obtained. He had been on 23 prescription medications including furosemide, metolazone, lisinopril and aspirin. Laboratory examination showed serum white blood cell count of 17.8, Blood Urea Nitrogen (BUN) of $50 \mathrm{mg} / \mathrm{dL}$, serum creatinine (Cr) of $7.1 \mathrm{mg} / \mathrm{dL}$, (according to KDIGO stage 2 renal impairment with increment in serum creatinine 2.9 times of the baseline) serum calcium corrected for albumin was $9.5 \mathrm{mg} / \mathrm{dL}$ and serum lactic acid was elevated. Blood gases and chemistries returned with evidence of anion gap metabolic acidosis with compensatory respiratory response. The blood cultures showed gram positive cocci. Eventually group A beta-hemolytic streptococcus was recovered from both blood and urine cultures. He was resuscitated adequately on normal saline 3L bolus till his blood pressure improved to $110 / 70 \mathrm{mmHg}$. His stay in the hospital spanned duration of two months thereafter.

Most noticeably, in the first few days, there was a rapid decline in the patient's renal function, with BUN between 90 and $137 \mathrm{mg} /$ $\mathrm{dL}$ and creatinine 9.9 to $12.3 \mathrm{mg} / \mathrm{dL}$ with no urine output. The decline in renal function was attributed to volume depletion and acute tubular necrosis. Hemodialysis or continuous veno-venous hemofiltration was considered but there was no volume overload or uncorrected acidosis. The anuric period was only a few hours and his renal function began to slowly improve (Figure 1) without institution of dialysis. His serum calcium corrected for albumin

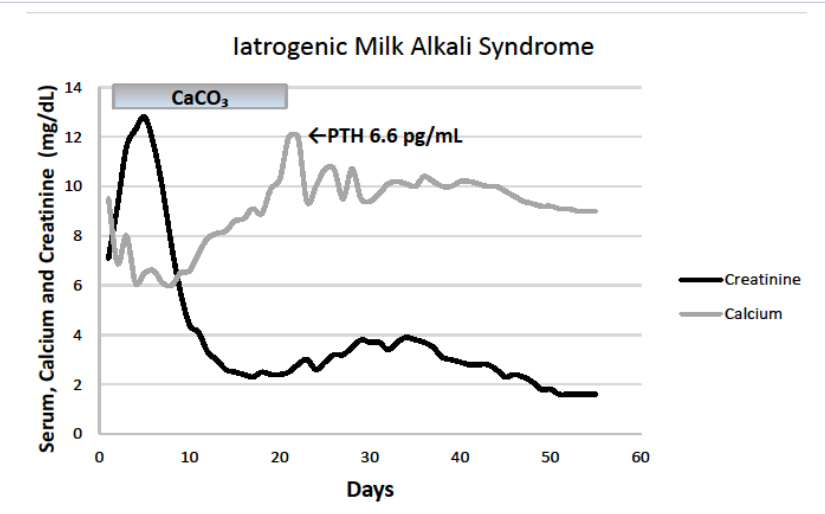

Figure 1: Graphical depiction of calcium and creatinine trends during and after calcium carbonate $\left(\mathrm{CaCO}_{3}\right)$. PTH (Serum Parathyroid Hormone) 
was $8.0 \mathrm{mg} / \mathrm{dL}$ and phosphorus rose to between 7.2 and 8.0 $\mathrm{mg} / \mathrm{dL}$ over the next week of his stay. In addition to ceftriaxone and clindamycin for the management of group A beta-hemolytic streptococcus bacteremia and intravenous fluid resuscitation, he was started on calcium carbonate suspension $500 \mathrm{mg} / 5 \mathrm{ml} 30 \mathrm{ml}$ every 3 hours, on the second day of his admission, as treatment for the marked hyperphosphatemia. He was also started on calcium carbonate tablets 3900 mg TID with each meal on the 6th day of his stay. His urine output improved progressively over the next 10 days with BUN and Cr trending down but still high in comparison to the normal range. He was transferred from the medical intensive care unit to an internal medicine bed on the 20th day of his stay. At transfer his serum calcium was 12.0 $\mathrm{mg} / \mathrm{dL}$ with BUN 37 and $\mathrm{Cr}$ of 2.5 and high serum phosphorus associated with renal failure (See Figure 1). We noted that he was still consuming high doses of calcium carbonate even though his serum calcium corrected for albumin was quite elevated. A serum PTH was suppressed at a time when the serum calcium was elevated (Figure 1). He had thus developed iatrogenic milk alkali syndrome when high dose calcium carbonate therapy was continued despite recovery of renal function. Consequently his calcium carbonate was discontinued.

During the following month the patient's renal function fluctuated as he recovered from the septicemia he developed as a result of urinary tract infection with non-hemolytic staphylococci. His renal function eventually trended and improved close to his baseline. His BUN was $28 \mathrm{mg} / \mathrm{dL}$, serum $\mathrm{Cr}$ was $1.8 \mathrm{mg} / \mathrm{dL}$ and serum calcium corrected for albumin was $9.5 \mathrm{mg} / \mathrm{dL}$ with phosphorus of $5.5 \mathrm{mg} / \mathrm{dL}$ at discharge.

\section{Discussion}

In 1915 Bertram Sippy's rigorous yet straightforward treatment of Peptic Ulcer Disease (PUD) with dairy sources of calcium mixed with alkaline powders eventually lead to undesired consequences [1] (Figure 2). Although more than two thousand years ago Hippocrates [2] first described the adverse reactions to the ingestion of calcium with alkali, it was Hardt and River's, [3] who studied the toxic effect of the Sippy regimen on the renal function of patients. They also reasoned that patients with preexisting renal ailments were more susceptible to develop severe toxic manifestations with the then suggested PUD treatment [3]. Cope in 1936 observed a rise in serum calcium levels in addition to the toxic effects and described the well-known manifestations of milk alkali syndrome; namely, hypercalcemia, metabolic alkalosis and renal insufficiency [4]. Cope also noted that the symptoms and renal function returns to normal once the

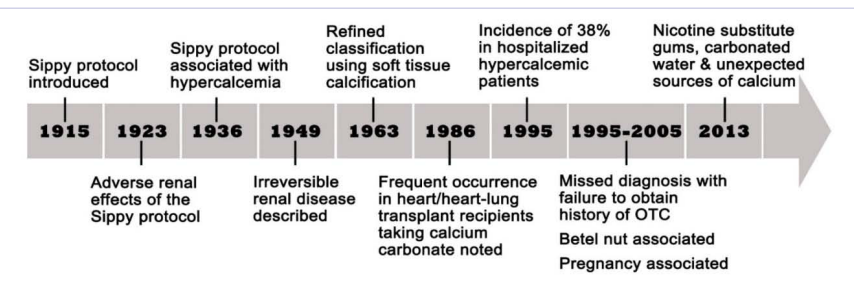

Figure 2: Timeline of the milk-alkali syndrome. treatment was held. However, in 1949 Burnett and colleagues described a more chronic course, with irreversible renal failure and metastatic calcification [5]. It was only after the institution of histamine- 2 blockers and non-absorbable antacids in the management of peptic ulcer disease that the incidence of milk alkali syndrome started to decline. By 1985 milk alkali syndrome was found be the cause of less than $1 \%$ of hypercalcemia [6].

Since 1982 there has been an increase in the diagnosis of milk alkali syndrome [6]. Several case series have documented that milk alkali is now common among patients hospitalized with hypercalcemia as well as in outpatients typically prescribed high dose calcium carbonate [6-8]. This rise is observed with many changes related to etiology and demographics [6-8]. The source of calcium in the Sippy regimen was milk, which also contains high phosphorus amounts. The source of calcium in today's milk alkali syndrome is calcium carbonate [2]. Hence, there has been a debate for this ailment to be named calcium alkali syndrome as the "milk" in milk alkali syndrome is not the principal etiologic source today [9].

Therefore, the classic MAS was the consequence of then suggested treatment for PUD. The modern MAS are more related to injudicious use of calcium carbonate as preventive measures for osteoporosis or stress ulcer prophylaxis in therapeutically immunosuppressed patients. There are many over-the-counter medicines that contain calcium in their formulation and can be additional sources of calcium for patients who are consuming calcium carbonate. As a consequence of the change from milk to calcium carbonate as the source of calcium in milk alkali syndrome, the demographic risk of developing this syndrome has shifted in the favor of women on calcium carbonate for osteoporosis prophylaxis, heart transplant patients and pregnant women $[6-8,10]$.

The pathophysiology of milk alkali syndrome, though not definitively clarified, can be considered a self-propagating vicious cycle starting with excessive calcium and absorbable alkali intake. Hypercalcemia has a direct effect on the renal function [2]. High calcium levels cause constriction of the renal afferent arterioles that leads to decreased glomerular filtration rate and creatinine clearance, which causes further increasing serum calcium levels. In addition to the effect on the glomeruli, hypercalcemia increases the renal tubular excretion of sodium that ultimately results in extracellular volume contraction. Presence of alkalosis in itself is a complicated and multifactorial aspect of the syndrome [2]. The volume depletion due to hypercalcemia leads to increased reabsorption of bicarbonate from the proximal renal tubule. As a consequence of the negative feedback mechanism, hypercalcemia leads to a decrease in serum parathyroid hormone. PTH deceases reabsorption of bicarbonate; hence low levels of the hormone promote bicarbonate accumulation, which further leads to a decrease in the PTH levels. It is now definitively known that PTH is low in MAS. These pat-ophysiologic mechanisms ultimately lead to calcium and alkali increases resulting in hypercalcemia, metabolic alkalosis and renal function impairment.

Many case reports have been published in the past few years that describe very unusual sources of calcium in addition to calcium carbonate as an etiology of milk alkali syndrome. 
Swanson et al, reported a 64 year old woman presenting with recurrent hypercalcemia [11]. The patient was diagnosed with milk alkali syndrome and the source of her calcium was found to be nicotine replacement gums and carbonated water. Fung, et al, [12] presented a 57 year old woman with an extensive past medical history including chronic kidney disease and resection of neuroendocrine tumor. Their patient was repeatedly admitted with unexplained intermittent hypercalcemia, which eventually was found to result from their patient's antacid consumption. Two important conclusions can be drawn from these case reports. Firstly, the importance of detailed history taking is of paramount significance when a patient presents with or develops hypercalcemia during stay in the hospital. Inquiries regarding intake of medicines, brand names, doses and uncommon sources of calcium have to be made in order to diagnose MAS. Secondly, as Fung et al, [12] have stressed in their case report, patients with pre-existing chronic ailments like chronic kidney disease may mask the laboratory findings of metabolic alkalosis. The suspicion threshold should be even lower in such patients in order to determine the etiology of hypercalcemia.

Our patient's scenario is unique because he did not present with hypercalcemia, he developed hypercalcemia during the course of his stay in the inpatient facility. He developed iatrogenic milk alkali syndrome with high serum calcium, impaired renal function, metabolic alkalosis and the patient improved when calcium carbonate was held. His serum PTH level was $6.6 \mathrm{pg} / \mathrm{dL}$, which substantiates the diagnosis. The utilization of intact parathyroid hormone assay even in patients with renal failure has made distinction of hypercalcemia as a PTH-dependent or -independent entity relatively easy [2]. Our patient was not worked up for hypercalcemia in depth as he was not hypercalcemic on presentation, and hypercalcemia resolved with stopping calcium carbonate. This case report demonstrates the requirement of observance from the participating physicians to ensure timely diagnosis and treatment of MAS.

\section{Conclusion}

Milk alkali syndrome has seen a historic past (Figure 2), etiologic shifts and demographic changes. The imbalance still presents as hypercalcemia, metabolic alkalosis, and renal impairment and happens to be the third most common cause of hypercalcemia in patients being admitted to the hospital [6]. The diagnosis however can be potentially missed if detailed history of calcium and alkali intake is not elicited from the patient. Those with a co-existing disease seem to be more at risk. The treatment is to remove the source of exogenous calcium and future education of the patient about calcium consumption.

\section{Acknowledgements}

This work was supported in part by NIH grants AR053483, AI082714 and GM104938.

\section{References}

1. Sippy BW. Gastric and duodenal ulcer. Medical cure by an efficient removal of gastric juice corrosion. JAMA.1983;250(16):2192-7.

2. Beall DP, Henslee HB, Webb HR, Scofield RH.. Milk-alkali syndrome: a historical review and description of the modern version of the syndrome. Am J Med Sci. 2006;331(5):233-42.

3. Hardt LL RA. Toxic manifestations following the alkaline treatment of peptic ulcer. Arch Internal medicine 1923;31(2):171-180. doi:10.1001/archinte.1923.00110140023003.

4. Cope CL. Base changes in alkalosis produced by the treatment of gastric ulcer with alkalies. Clinical Science.1936;2:13.

5. Burnett $\mathrm{CH}$, Commons RR, Albright F, et al. Hypercalcemia without Hypercalcuria or Hypophosphatemia, Calcinosis and Renal Insufficiency. New England Journal of Medicine 1949;240(20):787-94.

6. Beall DP, Scofield RH. Milk-alkali syndrome associated with calcium carbonate consumption. Report of7 patients with parathyroid hormone levels and an estimate of prevalence among patients hospitalized with hypercalcemia. Medicine (Baltimore) 1995;74(2):89-96.

7. Picolos MK, Lavis VR, Orlander PR. Milk-alkali syndrome is a major cause of hypercalcaemia among non-end-stage renal disease (nonESRD) inpatients. Clin Endocrinol (Oxf). 2005;63(5):566-76.

8. Kapsner P, Langsdorf L, Marcus R, Kraemer FB, Hoffman AR. Milkalkali syndrome in patients treated with calcium carbonate after cardiac transplantation. Arch Intern Med. 1986;146(10):1965-8.

9. Patel AM, Goldfarb S. Got calcium? Welcome to the calcium-alkali syndrome. J Am Soc Nephrol. 2010;21(9):1440-3. doi: 10.1681/ ASN.2010030255.

10. Kolnick L, Harris BD, Choma DP, Choma NN. Hypercalcemia in pregnancy: a case of milk-alkali syndrome. J Gen Intern Med. 2011;26(8):939-42. doi: 10.1007/s11606-011-1658-0.

11. Swanson CM, Mackey PA, Westphal SA, Argueta R. Nicotine-substitute gum-induced milk alkali syndrome: a look at unex-pected sources of calcium. Endocr Pract. 2013;19(6):142-4. doi: 10.4158/EP13080.CR.

12. Fung TY, Havekes B, Cornelis T. Milk-alkali syndrome sine alkalosis; an elusive cause of intermittent hypercalcemia. Int Urol Nephrol. 2013;45(6):1791-3. doi: 10.1007/s11255-012-0281-2. 